\title{
ORGANISATION EUROPÉENNE POUR LA RECHERCHE NUCLÉAIRE (CERN)
} Laboratoire Européen pour la Physique des Particules

CERN-EP/2000-139

13 November 2000

\section{Search for Supersymmetric Particles in $\mathrm{e}^{+} \mathrm{e}^{-}$Collisions at $\sqrt{s}$ up to $202 \mathrm{GeV}$ and Mass Limit for the Lightest Neutralino}

\author{
The ALEPH Collaboration*)
}

\begin{abstract}
Searches for pair production of squarks, sleptons, charginos and neutralinos are performed with the data collected by the ALEPH detector at LEP at centre-of-mass energies from 188.6 to $201.6 \mathrm{GeV}$. No evidence for any such signals is observed in a total integrated luminosity of about $410 \mathrm{pb}^{-1}$. The negative results of the searches are translated into exclusion domains in the space of the relevant MSSM parameters, which improve significantly on the constraints set previously. Under the assumptions of gaugino and sfermion mass unification, these results allow a 95\% C.L. lower limit of $37 \mathrm{GeV} / c^{2}$ to be set on the mass of the lightest neutralino for any $\tan \beta$ and sfermion mass. Additional constraints in the MSSM parameter space are derived from the negative results of ALEPH searches for Higgs bosons. The results are also interpreted in the framework of minimal supergravity.
\end{abstract}

To be submitted to Physics Letters $B$

*) See next pages for the list of authors 


\section{The ALEPH Collaboration}

R. Barate, I. De Bonis, D. Decamp, P. Ghez, C. Goy, S. Jezequel, J.-P. Lees, F. Martin, E. Merle, M.-N. Minard, B. Pietrzyk

Laboratoire de Physique des Particules (LAPP), IN²P3-CNRS, F-74019 Annecy-le-Vieux Cedex, France

S. Bravo, M.P. Casado, M. Chmeissani, J.M. Crespo, E. Fernandez, M. Fernandez-Bosman, Ll. Garrido, ${ }^{15}$ E. Graugés, J. Lopez, M. Martinez, G. Merino, R. Miquel, Ll.M. Mir, A. Pacheco, D. Paneque, H. Ruiz Institut de Física d'Altes Energies, Universitat Autònoma de Barcelona, E-08193 Bellaterra (Barcelona), Spain ${ }^{7}$

A. Colaleo, D. Creanza, N. De Filippis, M. de Palma, G. Iaselli, G. Maggi, M. Maggi, ${ }^{1}$ S. Nuzzo, A. Ranieri, G. Raso, ${ }^{24}$ F. Ruggieri, G. Selvaggi, L. Silvestris, P. Tempesta, A. Tricomi, ${ }^{3}$ G. Zito

Dipartimento di Fisica, INFN Sezione di Bari, I-70126 Bari, Italy

X. Huang, J. Lin, Q. Ouyang, T. Wang, Y. Xie, R. Xu, S. Xue, J. Zhang, L. Zhang, W. Zhao

Institute of High Energy Physics, Academia Sinica, Beijing, The People's Republic of China ${ }^{8}$

D. Abbaneo, P. Azzurri, T. Barklow, ${ }^{30}$ G. Boix,${ }^{6}$ O. Buchmüller, M. Cattaneo, F. Cerutti, B. Clerbaux, G. Dissertori, H. Drevermann, R.W. Forty, M. Frank, F. Gianotti, T.C. Greening, J.B. Hansen, J. Harvey, D.E. Hutchcroft, P. Janot, B. Jost, M. Kado, V. Lemaitre, P. Maley, P. Mato, A. Minten, A. Moutoussi, F. Ranjard, L. Rolandi, D. Schlatter, M. Schmitt, ${ }^{20}$ O. Schneider, ${ }^{2}$ P. Spagnolo, W. Tejessy, F. Teubert, E. Tournefier, ${ }^{26}$ A. Valassi, J.J. Ward, A.E. Wright

European Laboratory for Particle Physics (CERN), CH-1211 Geneva 23, Switzerland

Z. Ajaltouni, F. Badaud, S. Dessagne, A. Falvard, D. Fayolle, P. Gay, P. Henrard, J. Jousset, B. Michel, S. Monteil, J-C. Montret, D. Pallin, J.M. Pascolo, P. Perret, F. Podlyski

Laboratoire de Physique Corpusculaire, Université Blaise Pascal, IN ${ }^{2} P^{3}{ }_{-} C N R S$, Clermont-Ferrand, F-63177 Aubière, France

J.D. Hansen, J.R. Hansen, P.H. Hansen, B.S. Nilsson, A. Wäänänen

Niels Bohr Institute, 2100 Copenhagen, DK-Denmark ${ }^{9}$

G. Daskalakis, A. Kyriakis, C. Markou, E. Simopoulou, A. Vayaki

Nuclear Research Center Demokritos (NRCD), GR-15310 Attiki, Greece

A. Blondel, ${ }^{12}$ J.-C. Brient, F. Machefert, A. Rougé, M. Swynghedauw, R. Tanaka H. Videau

Laboratoire de Physique Nucléaire et des Hautes Energies, Ecole Polytechnique, IN ${ }^{2} P^{3}-C N R S$,

F-91128 Palaiseau Cedex, France

E. Focardi, G. Parrini, K. Zachariadou

Dipartimento di Fisica, Università di Firenze, INFN Sezione di Firenze, I-50125 Firenze, Italy

A. Antonelli, M. Antonelli, G. Bencivenni, G. Bologna, ${ }^{4}$ F. Bossi, P. Campana, G. Capon, V. Chiarella, P. Laurelli, G. Mannocchi, ${ }^{5}$ F. Murtas, G.P. Murtas, L. Passalacqua, M. Pepe-Altarelli ${ }^{25}$

Laboratori Nazionali dell'INFN (LNF-INFN), I-00044 Frascati, Italy

M. Chalmers, A.W. Halley, J. Kennedy, J.G. Lynch, P. Negus, V. O’Shea, B. Raeven, D. Smith, P. Teixeira-Dias, A.S. Thompson

Department of Physics and Astronomy, University of Glasgow, Glasgow G12 8QQ,United Kingdom ${ }^{10}$

R. Cavanaugh, S. Dhamotharan, C. Geweniger, P. Hanke, V. Hepp, E.E. Kluge, G. Leibenguth, A. Putzer, K. Tittel, S. Werner, ${ }^{19}$ M. Wunsch ${ }^{19}$

Kirchhoff-Institut für Physik, Universität Heidelberg, D-69120 Heidelberg, Germany ${ }^{16}$ 
R. Beuselinck, D.M. Binnie, W. Cameron, G. Davies, P.J. Dornan, M. Girone, ${ }^{1}$ N. Marinelli, J. Nowell, H. Przysiezniak, J.K. Sedgbeer, J.C. Thompson, ${ }^{14}$ E. Thomson, ${ }^{23}$ R. White

Department of Physics, Imperial College, London SW7 2BZ, United Kingdom ${ }^{10}$

V.M. Ghete, P. Girtler, E. Kneringer, D. Kuhn, G. Rudolph

Institut für Experimentalphysik, Universität Innsbruck, A-6020 Innsbruck, Austria ${ }^{18}$

E. Bouhova-Thacker, C.K. Bowdery, D.P. Clarke, G. Ellis, A.J. Finch, F. Foster, G. Hughes, R.W.L. Jones, ${ }^{1}$ M.R. Pearson, N.A. Robertson, M. Smizanska

Department of Physics, University of Lancaster, Lancaster LA1 4YB, United Kingdom ${ }^{10}$

I. Giehl, F. Hölldorfer, K. Jakobs, K. Kleinknecht, M. Kröcker, A.-S. Müller, H.-A. Nürnberger, G. Quast, ${ }^{1}$

B. Renk, E. Rohne, H.-G. Sander, S. Schmeling, H. Wachsmuth, C. Zeitnitz, T. Ziegler

Institut für Physik, Universität Mainz, D-55099 Mainz, Germany ${ }^{16}$

A. Bonissent, J. Carr, P. Coyle, C. Curtil, A. Ealet, D. Fouchez, O. Leroy, T. Kachelhoffer, P. Payre, D. Rousseau, A. Tilquin

Centre de Physique des Particules de Marseille, Univ Méditerranée, $I N^{2} P^{3}$-CNRS, F-13288 Marseille, France

M. Aleppo, S. Gilardoni, F. Ragusa

Dipartimento di Fisica, Università di Milano e INFN Sezione di Milano, I-20133 Milano, Italy.

A. David, H. Dietl, G. Ganis, ${ }^{27}$ A. Heister, K. Hüttmann, G. Lütjens, C. Mannert, W. Männer, H.-G. Moser, S. Schael, R. Settles, ${ }^{1}$ H. Stenzel, G. Wolf

Max-Planck-Institut für Physik, Werner-Heisenberg-Institut, D-80805 München, Germany ${ }^{16}$

J. Boucrot, ${ }^{1}$ O. Callot, M. Davier, L. Duflot, J.-F. Grivaz, Ph. Heusse, A. Jacholkowska, ${ }^{1}$ L. Serin, J.-J. Veillet, I. Videau, J.-B. de Vivie de Régie, ${ }^{28}$ C. Yuan, D. Zerwas

Laboratoire de l'Accélérateur Linéaire, Université de Paris-Sud, $I N^{2} P^{3}$-CNRS, F-91898 Orsay Cedex, France

G. Bagliesi, T. Boccali, G. Calderini, V. Ciulli, L. Foà, A. Giammanco, A. Giassi, F. Ligabue, A. Messineo, F. Palla, ${ }^{1}$ G. Sanguinetti, A. Sciabà, G. Sguazzoni, R. Tenchini, ${ }^{1}$ A. Venturi, P.G. Verdini

Dipartimento di Fisica dell'Università, INFN Sezione di Pisa, e Scuola Normale Superiore, I-56010

Pisa, Italy

G.A. Blair, J. Coles, G. Cowan, M.G. Green, L.T. Jones, T. Medcalf, J.A. Strong Department of Physics, Royal Holloway \& Bedford New College, University of London, Surrey TW20 OEX, United Kingdom ${ }^{10}$

R.W. Clifft, T.R. Edgecock, P.R. Norton, I.R. Tomalin

Particle Physics Dept., Rutherford Appleton Laboratory, Chilton, Didcot, Oxon OX11 OQX, United Kingdom ${ }^{10}$

B. Bloch-Devaux, ${ }^{1}$ D. Boumediene, P. Colas, B. Fabbro, E. Lançon, M.-C. Lemaire, E. Locci, P. Perez, J. Rander, J.-F. Renardy, A. Rosowsky, P. Seager, ${ }^{13}$ A. Trabelsi, ${ }^{21}$ B. Tuchming, B. Vallage

CEA, DAPNIA/Service de Physique des Particules, CE-Saclay, F-91191 Gif-sur-Yvette Cedex, France $^{17}$

N. Konstantinidis, C. Loomis, A.M. Litke, G. Taylor

Institute for Particle Physics, University of California at Santa Cruz, Santa Cruz, CA 95064, USA 22

C.N. Booth, S. Cartwright, F. Combley, P.N. Hodgson, M. Lehto, L.F. Thompson

Department of Physics, University of Sheffield, Sheffield S3 7RH, United Kingdom ${ }^{10}$

K. Affholderbach, A. Böhrer, S. Brandt, C. Grupen, J. Hess, A. Misiejuk, G. Prange, U. Sieler

Fachbereich Physik, Universität Siegen, D-57068 Siegen, Germany ${ }^{16}$

C. Borean, G. Giannini, B. Gobbo

Dipartimento di Fisica, Università di Trieste e INFN Sezione di Trieste, I-34127 Trieste, Italy 
H. He, J. Putz, J. Rothberg, S. Wasserbaech

Experimental Elementary Particle Physics, University of Washington, Seattle, WA 98195 U.S.A.

S.R. Armstrong, K. Cranmer, P. Elmer, D.P.S. Ferguson, Y. Gao ${ }^{29}$ S. González, O.J. Hayes, H. Hu, S. Jin, J. Kile, P.A. McNamara III, J. Nielsen, W. Orejudos, Y.B. Pan, Y. Saadi, I.J. Scott, J.H. von Wimmersperg-Toeller, J. Walsh, W. Wiedenmann, J. Wu, Sau Lan Wu, X. Wu, G. Zobernig

Department of Physics, University of Wisconsin, Madison, WI 53706, USA ${ }^{11}$

\footnotetext{
${ }^{1}$ Also at CERN, 1211 Geneva 23, Switzerland.

${ }^{2}$ Now at Université de Lausanne, 1015 Lausanne, Switzerland.

${ }^{3}$ Also at Dipartimento di Fisica di Catania and INFN Sezione di Catania, 95129 Catania, Italy.

${ }^{4}$ Deceased.

${ }^{5}$ Also Istituto di Cosmo-Geofisica del C.N.R., Torino, Italy.

${ }^{6}$ Supported by the Commission of the European Communities, contract ERBFMBICT982894.

${ }^{7}$ Supported by CICYT, Spain.

${ }^{8}$ Supported by the National Science Foundation of China.

${ }^{9}$ Supported by the Danish Natural Science Research Council.

${ }^{10}$ Supported by the UK Particle Physics and Astronomy Research Council.

${ }^{11}$ Supported by the US Department of Energy, grant DE-FG0295-ER40896.

${ }^{12}$ Now at Departement de Physique Corpusculaire, Université de Genève, 1211 Genève 4, Switzerland.

${ }^{13}$ Supported by the Commission of the European Communities, contract ERBFMBICT982874.

${ }^{14}$ Also at Rutherford Appleton Laboratory, Chilton, Didcot, UK.

${ }^{15}$ Permanent address: Universitat de Barcelona, 08208 Barcelona, Spain.

${ }^{16}$ Supported by the Bundesministerium für Bildung, Wissenschaft, Forschung und Technologie,

${ }^{17}$ Supported by the Direction des Sciences de la Matière, C.E.A.

${ }^{18}$ Supported by the Austrian Ministry for Science and Transport.

${ }^{19}$ Now at SAP AG, 69185 Walldorf, Germany

${ }^{20}$ Now at Harvard University, Cambridge, MA 02138, U.S.A.

${ }^{21}$ Now at Département de Physique, Faculté des Sciences de Tunis, 1060 Le Belvédère, Tunisia.

${ }^{22}$ Supported by the US Department of Energy, grant DE-FG03-92ER40689.

${ }^{23}$ Now at Department of Physics, Ohio State University, Columbus, OH 43210-1106, U.S.A.

${ }^{24}$ Also at Dipartimento di Fisica e Tecnologie Relative, Università di Palermo, Palermo, Italy.

${ }^{25}$ Now at CERN, 1211 Geneva 23, Switzerland.

${ }^{26}$ Now at ISN, Institut des Sciences Nucléaires, 53 Av. des Martyrs, 38026 Grenoble, France.

${ }^{27}$ Now at INFN Sezione di Roma II, Dipartimento di Fisica, Università di Roma Tor Vergata, 00133 Roma, Italy.

${ }^{28}$ Now at Centre de Physique des Particules de Marseille,Univ Méditerranée, F-13288 Marseille, France.

${ }^{29}$ Also at Department of Physics, Tsinghua University, Beijing, The People's Republic of China.

${ }^{30}$ Also at SLAC, Stanford, CA 94309, U.S.A.
} Germany. 


\section{Introduction}

In this letter the results of searches for sleptons $(\widetilde{\ell})$, squarks $(\widetilde{q})$, charginos $\left(\chi^{ \pm}, \chi_{2}^{ \pm}\right)$and neutralinos $\left(\chi_{i}^{0}\right)$ are reported, obtained with the data collected by the ALEPH detector at LEP during 1999 (for squark and slepton searches) and 1998-1999 (for chargino and neutralino searches), at centre-of-mass energies ranging from 188.6 to 201.6 GeV. Energies and integrated luminosities of the analysed data samples are given in Table 11. Results of slepton and squark searches with the 1998 data sample have already been reported in Ref. [1]. As in Refs. [1, 2, 3], the theoretical framework is the Minimal Supersymmetric extension of the Standard Model (MSSM), with R-parity conservation and the assumption that the lightest neutralino is the Lightest Supersymmetric Particle (LSP). The notations and conventions described in Ref. [2] are used for the MSSM parameters.

Table 1: Definition of the analysed data samples.

\begin{tabular}{|l||c||c|c|c|c|}
\hline \multicolumn{1}{|c||}{} & 1998 & \multicolumn{4}{c|}{1999} \\
\hline$\sqrt{s}(\mathrm{GeV})$ & 188.6 & 191.6 & 195.5 & 199.5 & 201.6 \\
$\int \mathcal{L} \mathrm{d} t\left(\mathrm{pb}^{-1}\right)$ & 174.2 & 28.9 & 79.8 & 86.2 & 42.0 \\
\hline
\end{tabular}

The final state topologies addressed by the searches are summarized in Table 2 together with the related signal processes. For a given final state topology, various selection criteria are applied which depend mainly on the mass difference $\Delta M$ between the produced particle and the LSP. The selection algorithms are basically the same as in previous publications [1, 2, 3] but, in order to cope with the increased centre-of-mass energy and with the larger size of the new data samples, the positions of the cuts were re-optimized so as to give the lowest expected upper limit on the number of produced signal events in the case of absence of signal.

For the interpretation of the results in the MSSM, the unification relation among the gaugino supersymmetry breaking mass terms, $M_{1}=\frac{5}{3} M_{2} \tan ^{2} \theta_{W}$, is assumed. The region where $M_{2} \gg|\mu|$ is referred to as the higgsino region, and the region where $|\mu| \gg M_{2}$ as the gaugino region. Unless otherwise specified, all supersymmetric particle masses and couplings are calculated at tree level. For charginos and neutralinos, they are entirely determined by $M_{2}$, the Higgs mass term $\mu$, and the ratio of the vacuum expectation values of the two Higgs doublets, commonly indicated with $\tan \beta$. When relevant, the sfermion masses are calculated from the renormalization group equations assuming a common supersymmetry breaking mass term $m_{0}$ for all sleptons and squarks at the grandunification scale. The results in the gaugino sector, in particular the LSP mass lower limit, are derived under the assumption of flavour-independent leptonic branching ratios. The possible impact on these results of a large mixing in the stau sector is therefore not considered in this letter.

The results of Higgs boson searches [4 are used to further constrain the MSSM parameter space as discussed in Ref. [3]. The inclusion of the Higgs boson searches requires that the pseudo-scalar neutral Higgs boson mass, $m_{\mathrm{A}}$, and the trilinear coupling in the 
Table 2: Topologies studied in the different searches; only the main decay chains contributing to the different topologies are indicated for neutralinos.

\begin{tabular}{|l|l|l|}
\hline Production & Decay mode & Topology \\
\hline \hline$\widetilde{\ell} \widetilde{\ell}$ & $\widetilde{\ell} \rightarrow \ell \chi_{1}^{0}$ & Acoplanar leptons \\
$\widetilde{\mathrm{e}}_{\mathrm{L}(\mathrm{R})} \overline{\tilde{\mathrm{e}}}_{\mathrm{R}(\mathrm{L})}$ & $\widetilde{\mathrm{e}} \rightarrow \mathrm{e} \chi_{1}^{0}$ & Single electron (small $\left.m_{\widetilde{\mathrm{e}}_{\mathrm{R}}}-m_{\chi_{1}^{0}}\right)$ \\
\hline \hline$\widetilde{\mathrm{q}} \overline{\mathrm{q}}$ & $\widetilde{\mathrm{q}} \rightarrow \mathrm{q} \chi_{1}^{0}$ & Acoplanar jets \\
$\widetilde{\mathrm{t}}$ & $\widetilde{\mathrm{t}} \rightarrow \mathrm{c} \chi_{1}^{0}$ & Acoplanar jets \\
$\widetilde{\mathrm{b}} \overline{\mathrm{b}}$ & $\widetilde{\mathrm{b}} \rightarrow \mathrm{b} \chi_{1}^{0}$ & Acoplanar b-jets \\
$\widetilde{\mathrm{t}}$ & $\widetilde{\mathrm{t}} \rightarrow \mathrm{b} \ell \widetilde{\nu}$ & Acoplanar jets plus leptons \\
\hline \hline$\chi^{+} \chi^{-}$ & $\chi^{ \pm} \rightarrow \mathrm{q} \overline{\mathrm{q}}^{\prime} \chi_{1}^{0}$ & 4 jets $+\not E$ \\
& $\chi^{ \pm} \rightarrow \ell^{ \pm} \nu \chi_{1}^{0}$ & Acoplanar leptons \\
& mixed & 2 jets + lepton $+\not E$ \\
\hline \hline$\chi_{i}^{0} \chi_{j}^{0}$ & $\chi_{1}^{0} \chi_{j}^{0} \rightarrow \mathrm{q} \overline{\mathrm{q}} \chi_{1}^{0}$ & Acoplanar jets \\
& $\chi_{i}^{0} \chi_{j}^{0} \rightarrow \nu \bar{\nu} \chi_{1}^{0} q \overline{\mathrm{q}} \chi_{1}^{0}, \ldots$ & \\
$j \geq i, j \neq 1$ & $\chi_{1}^{0} \chi_{j}^{0} \rightarrow \ell^{+} \ell^{-} \chi_{1}^{0}$ & Acoplanar leptons \\
& $\chi_{i}^{0} \chi_{j}^{0} \rightarrow \nu \bar{\nu} \chi_{1}^{0} \ell^{+} \ell^{-} \chi_{1}^{0}, \ldots$ & \\
\hline
\end{tabular}

stop sector, $A_{\mathrm{t}}$, be included in the analysis. Masses and mixing angles in the Higgs sector are obtained with the two-loop level calculations of Ref. [5], as implemented in Ref. [6].

As in Ref. [3], the results are also interpreted in the framework of a highly constrained MSSM version known as minimal supergravity. Masses and couplings are calculated in terms of five parameters: the mass term $m_{0}$ common to all scalars (Higgs bosons, squarks and sleptons), the common supersymmetry-breaking gaugino mass term $m_{1 / 2}$, and a common trilinear coupling $A_{0}$ (all defined at the grand unification scale), $\tan \beta$ and the sign of $\mu$. To solve the appropriate set of renormalization group equations, the latest version of the ISAJET package [0] is used. For this analysis, the one-loop radiative corrections to chargino and neutralino masses [8] are included.

This letter is organized as follows. The modifications to the selection algorithms and the results of the searches are described in Section 2. The interpretation of these results in the theoretical frameworks mentioned above is presented in Section 3, with a special focus on the lower limit on the LSP mass. The conclusions are given in Section 4 .

A thorough description of the ALEPH detector and of its performance, as well as of the standard reconstruction and analysis algorithms, can be found in Refs. [9, 10]. Only a brief summary is given here. Charged particle tracking, down to $16^{\circ}$ from the beam axis, is obtained by means of a silicon vertex detector, a cylindrical drift chamber, and a large time projection chamber, all immersed in a $1.5 \mathrm{~T}$ axial magnetic field provided by a superconducting solenoidal coil. Hermetic calorimetric coverage, down to polar angles of $34 \mathrm{mrad}$, is achieved by means of a highly granular electromagnetic calorimeter, by dedicated low angle luminosity monitors, and by the iron return yoke instrumented to act as a hadron calorimeter. The latter is supplemented with external muon chambers. The 
information from all these detectors is combined in an energy-flow algorithm [10] which provides a list of charged particles (electrons, muons, charged hadrons), photons and neutral hadrons, used also to determine global quantities such as total energy or missing momentum. The resolution achieved on the total visible energy is $(0.6 \sqrt{E}+0.6) \mathrm{GeV}(E$ in $\mathrm{GeV}$ ).

\section{Update of sparticle searches}

The simulation of the signal production, which includes a detailed treatment of cascade decays as well as of initial and final state radiation, was performed with SUSYGEN [11. For the determination of the selection efficiencies, a fast detector simulation, cross-checked with several fully simulated samples, was used.

Standard model processes were simulated with BHWIDE [12 for Bhabha production, KORALZ [13 for $\mu^{+} \mu^{-}$and $\tau^{+} \tau^{-}$production, PHOT02 14 for $\gamma \gamma \rightarrow$ leptons and tagged $\gamma \gamma \rightarrow$ hadrons, PHOJET [15] for untagged $\gamma \gamma \rightarrow$ hadrons, KORALW 16] for $\mathrm{W}^{+} \mathrm{W}^{-}$, Grace4F [17] for $\mathrm{We} \nu$, a private generator [18] for Z $\nu \bar{\nu}$ events, and PYTHIA [19] for all other processes. The integrated luminosity of the simulated samples corresponds to at least 25 times the integrated luminosity of the data sample, except for $\gamma \gamma$ processes for which the statistics are at least a factor of three larger than that of the data sample. All standard model background samples were processed through the full detector simulation.

Background subtraction was generally performed when optimizing the selection algorithms, except in searches for squarks and for hadronic and mixed final states arising from chargino production, where the expected gain is marginal.

\subsection{Update of sfermion searches}

The final states studied (Table 2) are those arising from squark and slepton pair-production followed by the decays $\widetilde{\mathrm{t}} \rightarrow \mathrm{c} \chi_{1}^{0}, \widetilde{\mathrm{t}} \rightarrow \mathrm{b} \ell \widetilde{\nu}, \widetilde{\mathrm{b}} \rightarrow \mathrm{b} \chi_{1}^{0}, \widetilde{\mathrm{q}} \rightarrow \mathrm{q} \chi_{1}^{0}, \widetilde{\mathrm{e}} \rightarrow \mathrm{e} \chi_{1}^{0}, \widetilde{\mu} \rightarrow \mu \chi_{1}^{0}$ and $\widetilde{\tau} \rightarrow \tau \chi_{1}^{0}$. Events with acoplanar jets and acoplanar jets plus two leptons are signatures for squark production. Events with acoplanar lepton pairs or with single electrons are expected from slepton production. All these final states are characterized by missing energy. To reach the best sensitivity to the expected signal for a large range of $\Delta M$ values, two different selection procedures are employed [1, 20], which specifically address the small and the large $\Delta M$ cases. Systematic uncertainties on selection efficiencies and background estimations remain at the few percent level, as in Ref. [1].

The numbers of events selected in the 1999 data by the sfermion searches are reported in Table 3, together with the results obtained with the 1998 data sample. In general, agreement is observed between numbers of candidate events and expectations from standard processes. However, a slight excess is observed in the acoplanar tau search; the probability for an upward statistical fluctuation of the expected background is $1.6 \%$ for the 1999 sample alone, and 1.2\% when the 1998 data are included. The events selected by the $\widetilde{b}$ searches are also selected by the $\widetilde{t} \rightarrow c \chi_{1}^{0}$ analyses. 


\section{$2.2 \quad$ Update of gaugino searches}

\section{Chargino searches}

Searches are performed in all possible chargino-pair decay topologies such as four-jets (4J), hadrons plus electron or muon $(2 \mathrm{~J} \ell)$, hadrons plus tau $(2 \mathrm{~J} \tau)$ and acoplanar lepton pairs $(\mathrm{A} \ell)$, as described in Refs. [2, 㓭. The selection efficiencies, the background contaminations and the main systematic uncertainties, related to the simulation of the energy-flow reconstruction and of the lepton identification, are similar to those reported for the analyses at lower energies. For each set of mass difference and leptonic branching ratio, the optimal combination of selections is determined as explained in Ref. [3].

\section{Neutralino searches}

In the case of large sfermion masses (large $m_{0}$ ), the neutralino searches are applied only in the higgsino region where the final state is characterized by acoplanar jets. The sensitivities of the two analyses, dedicated to small and large $\Delta M$, are significantly improved by subtracting the expected four-fermion background when re-optimizing the cuts. The selection efficiencies remain comparable to those of the analyses applied to the $183 \mathrm{GeV}$ data sample [3].

For small sfermion masses, the leptonic branching ratios are enhanced. A new search was developed to address acoplanar electron or muon final states. The selection consists of the preselection of the selectron and smuon searches supplemented with a final sliding cut on the sum of the two lepton energies. The exact location of this cut depends on the mass difference $\Delta M$ between the $\chi_{j}^{0}$ and the $\chi_{1}^{0}$. Selection efficiencies of $70 \%$ are reached for a large range of $\Delta M$ values, with an irreducible background of $0.37 \mathrm{pb}$, mainly due to $\mathrm{W}^{+} \mathrm{W}^{-} \rightarrow \ell^{+} \nu \ell^{-} \bar{\nu}$ events. The systematic uncertainties associated to this new selection are similar to those quoted for the selectron and smuon searches [四].

\section{Results}

The numbers of events observed and expected from standard processes for the chargino and neutralino selections are reported in Table 3. The numbers of candidate events are in agreement with the expected background for all the selections.

\section{$3 \quad$ Interpretation of the results}

As no significant excess of candidate events is observed, upper limits on the production cross sections are set for the processes searched for. Each candidate event contributes to a limited range of $\Delta M$. The systematic uncertainties on the selection efficiencies are included following the method described in Ref. [21]. When background subtraction is performed, the prescription of Ref. [22] is adopted, with the number of subtracted background events 
Table 3: Numbers of candidate events observed in the data $\left(N_{\text {cand }}\right)$ and background events expected from standard model processes $\left(N_{\mathrm{bkg}}\right)$.

\begin{tabular}{|c|c||c|c||c|c|}
\hline Process & \multicolumn{1}{|c||}{ Comment } & \multicolumn{2}{c||}{1998 data } & \multicolumn{2}{c|}{1999 data } \\
\cline { 3 - 6 } & & $N_{\text {cand }}$ & $N_{\text {bkg }}$ & $N_{\text {cand }}$ & $N_{\text {bkg }}$ \\
\hline$\widetilde{e} \widetilde{\mathrm{e}}$ & & 33 & 32.8 & 42 & 48.1 \\
\hline$\widetilde{\mu} \widetilde{\mu}$ & & 28 & 29.6 & 39 & 43.4 \\
\hline$\widetilde{\tau} \widetilde{\tau}$ & & 26 & 21.5 & 46 & 32.7 \\
\hline$\widetilde{\mathrm{e}}_{\mathrm{L}(\mathrm{R})} \overline{\mathrm{e}}_{\mathrm{R}(\mathrm{L})}$ & & 8 & 13.8 & 22 & 22.4 \\
\hline \hline$\widetilde{\mathrm{t}} \rightarrow \mathrm{c} \chi_{1}^{0}$ & small $\Delta M$ & 3 & 5.5 & 2 & 2.4 \\
& high $\Delta M$ & 5 & 4.0 & 8 & 7.3 \\
\hline$\widetilde{\mathrm{b}} \rightarrow \mathrm{b} \chi_{1}^{0}$ & small $\Delta M$ & 3 & 3.3 & 1 & 2.2 \\
& high $\Delta M$ & 0 & 0.9 & 1 & 0.7 \\
\hline$\widetilde{\mathrm{t}} \rightarrow \mathrm{b} / \widetilde{\nu}$ & small $\Delta M$ & 0 & 1.9 & 3 & 2.6 \\
& high $\Delta M$ & 2 & 0.4 & 2 & 1.4 \\
\hline \hline Chargino & W* branching ratios & 10 & 8.3 & 9 & 12.7 \\
& any branching ratios & 25 & 23.0 & 24 & 33.9 \\
\hline \hline Neutralino & Acop. Jets $\left(\Delta M<40 \mathrm{GeV} / c^{2}\right)$ & 4 & 3.0 & 6 & 4.5 \\
& Acop. Leptons & 59 & 60.0 & 76 & 79.8 \\
\hline
\end{tabular}

conservatively reduced by one standard deviation of its systematic uncertainty. Finally, the constraints presented in this section are derived by combining the searches presented here with those reported in Refs. [1, 2, 3], and are at 95\% confidence level.

\subsection{Slepton production}

The cross section upper limits, translated into excluded regions in the $\left(m_{\widetilde{\ell}_{\mathrm{R}}}, m_{\chi_{1}^{0}}\right)$ plane, are shown in Figs. 1 1a-c for $\tilde{\ell}_{\mathrm{R}}^{+} \widetilde{\ell}_{\mathrm{R}}^{-}$production and a $100 \% \tilde{\ell}_{\mathrm{R}} \rightarrow \ell \chi_{1}^{0}$ branching ratio. For selectrons, it is assumed that $\tan \beta=2$ and $\mu=-200 \mathrm{GeV} / c^{2}$. For $\widetilde{\mathrm{e}}$ and $\widetilde{\mu}$, the impact of cascade decays such as $\widetilde{\ell}_{\mathrm{R}} \rightarrow \ell \chi_{j}^{0}(j>1)$ is illustrated by the excluded region obtained with a vanishing selection efficiency for final states deriving from those decays. For $\Delta M>10 \mathrm{GeV} / c^{2}$, the lower limits on $m_{\widetilde{\mathrm{e}}_{\mathrm{R}}}, m_{\widetilde{\mu}_{\mathrm{R}}}$ and $m_{\widetilde{\tau}_{\mathrm{R}}}$ are 92,85 and $70 \mathrm{GeV} / c^{2}$, respectively. For staus, the exclusion obtained for the mixing angle corresponding to the minimal cross section is also shown. In such a case, the lower limit on the mass is $68 \mathrm{GeV} / c^{2}$ for $\Delta M>10 \mathrm{GeV} / c^{2}$.

The results of the searches for acoplanar leptons are combined with those of the search for events with single electrons, using the slepton masses determined from the GUT relations, and with the additional assumption of no mixing in the stau sector. The region of the plane $\left(m_{\widetilde{\ell}_{\mathrm{R}}}, m_{\chi_{1}^{0}}\right)$ excluded for $\tan \beta=2$ and $\mu=-200 \mathrm{GeV} / c^{2}$ is shown 
in Fig. 11d. The loss of sensitivity of the $\widetilde{\mathrm{e}}_{\mathrm{R}} \overline{\widetilde{\mathrm{e}}}_{\mathrm{R}}$ search for $\Delta M<3 \mathrm{GeV} / c^{2}$ is recovered for $m_{\tilde{\ell}}<70 \mathrm{GeV} / c^{2}$ by the search for $\widetilde{\mathrm{e}}_{\mathrm{R}}^{ \pm} \widetilde{\mathrm{e}}_{\mathrm{L}}^{\mp}$. The effect of an assumed null efficiency for cascade decays at small $m_{\chi_{1}^{0}}$ is compensated by the contribution of the $\tilde{\ell}_{\mathrm{L}} \tilde{\ell}_{\mathrm{L}}$ production.

\subsection{Squark production}

Under the assumption of a dominant $\widetilde{t} \rightarrow c \chi_{1}^{0}$ decay, the regions excluded by stop searches in the plane $\left(m_{\widetilde{t}}, m_{\chi_{1}^{0}}\right)$ are shown in Fig. 2a for two values of the $\widetilde{t}$ mixing angle $\theta_{\tilde{t}}, 0^{\circ}$ and $56^{\circ}$, corresponding to maximal and minimal cross section, respectively. For $\Delta M$ in the range from 6 to $40 \mathrm{GeV} / c^{2}$, i.e., a region not accessible to the Tevatron searches, the lower limit on $m_{\widetilde{t}}$ is $83 \mathrm{GeV} / c^{2}$, independent of $\theta_{\tilde{t}}$. In the case of a dominant $\tilde{t} \rightarrow \mathrm{b} \ell \widetilde{v}$ decay, the excluded region in the plane $\left(m_{\widetilde{\mathrm{t}}}, m_{\widetilde{\nu}}\right)$ is shown in Fig. 2 $\mathrm{b}$, where equal branching ratios for $\ell=\mathrm{e}, \mu$ and $\tau$ are assumed. For $\Delta M>10 \mathrm{GeV} / c^{2}$ and with the LEP 1 lower limit on the sneutrino mass of $43 \mathrm{GeV} / c^{2}$ (obtained for three mass degenerate $\tilde{\nu}$ 's), the $\theta_{\mathrm{t}}$-independent lower limit on $m_{\widetilde{\mathrm{t}}}$ is $88 \mathrm{GeV} / c^{2}$. The regions excluded by sbottom searches in the plane $\left(m_{\widetilde{\mathrm{b}}}, m_{\chi_{1}^{0}}\right)$ under the assumption of a dominant $\widetilde{\mathrm{b}} \rightarrow \mathrm{b} \chi_{1}^{0}$ decay are shown in Fig. Zc for two values of the $\widetilde{\mathrm{b}}$ mixing angle $\theta_{\widetilde{\mathrm{b}}}$, corresponding to minimal $\left(\theta_{\widetilde{\mathrm{b}}}=68^{\circ}\right)$ and maximal $\left(\theta_{\widetilde{\mathrm{b}}}=0^{\circ}\right)$ production cross section. In the latter case, and for $\Delta M>10 \mathrm{GeV} / c^{2}$, a lower limit of $91 \mathrm{GeV} / c^{2}$ is set on $m_{\widetilde{\mathrm{b}}}$.

In Fig. 2 $\mathrm{d}$, the negative outcome of the searches for acoplanar jets is translated into exclusion domains in the plane $\left(m_{\tilde{\mathrm{g}}}, m_{\tilde{\mathrm{q}}}\right)$ for mass-degenerate squarks (except the two stop particles) and with unification of the gluino and weak gaugino masses. Here $\tan \beta=4$ and $\mu=-400 \mathrm{GeV} / c^{2}$ are chosen, as in Refs. [23, 24].

The results obtained at the Tevatron [23, 24] are also shown in Fig. 2. The sensitivity of the squark searches presented in this letter extends to smaller $\Delta M$ values than those tested at hadron colliders.

\subsection{Gaugino production}

In Fig. 3a, the upper limit on the production cross section is displayed for chargino pairs with masses close to the kinematic limit at $\sqrt{s}=201.6 \mathrm{GeV}$. It is assumed that charginos decay through the process $\chi^{ \pm} \rightarrow \chi_{1}^{0} \mathrm{~W}^{ \pm *}$. In Figs. $3 \mathrm{~b}$-d $\mathrm{d}$, the gaugino cross section upper limits are translated, for $\tan \beta=\sqrt{2}$ and large $m_{0}$, into exclusion domains in the $\left(\mu, M_{2}\right)$ plane of the MSSM, into lower limits on the chargino mass, and into lower limits on $m_{\chi_{1}^{0}}+m_{\chi_{2}^{0}}$. The kinematic limit is closely approached for both chargino and associated neutralino production, except in the deep higgsino region in which $\Delta M$, and hence the selection efficiencies, are small. For $\tan \beta=\sqrt{2}$ and negative $\mu$, the indirect chargino mass limit derived from the neutralino searches extends beyond the chargino kinematic limit by up to $5 \mathrm{GeV} / c^{2}$. 


\subsection{Lower limit on the LSP mass}

From the negative outcome of chargino and neutralino searches, a lower limit on the mass of the lightest neutralino can be derived as a function of $\tan \beta$, as shown in Fig. 4 for $m_{0}=500 \mathrm{GeV} / c^{2}$. Neutralino searches contribute mostly at low $\tan \beta$ and play an essential rôle in determining the lowest value of $37.2 \mathrm{GeV} / c^{2}$, obtained for $\tan \beta=1$, $M_{2} \simeq 62 \mathrm{GeV} / c^{2}$ and $\mu \simeq-72 \mathrm{GeV} / c^{2}$, which remains valid also for larger $m_{0}$ values. For smaller $m_{0}$ values, the loss of sensitivity of chargino and neutralino searches is recovered by slepton searches, as discussed in Ref. [3]. A scan performed over the relevant parameter space, including the deep gaugino region for large $\tan \beta$ and for mass degenerate charginos and sneutrinos, shows that the lower limit on the LSP mass of $37.2 \mathrm{GeV} / c^{2}$, obtained for $\tan \beta=1$ at large $m_{0}$, holds for all values of $m_{0}$.

As discussed in Ref. [8], radiative corrections to chargino and neutralino masses modify the relation between such masses and therefore the interplay between chargino and neutralino searches. In the region where the limit is found, these corrections lower the LSP mass limit value by about $1 \mathrm{GeV} / c^{2}$. A further source of theoretical uncertainty is represented by the GUT relation between $M_{1}$ and $M_{2}$. Higher-order corrections to the one-loop formula used here have been estimated with ISAJET [7] to be of the order of $\pm 3 \%$, and affect by the same amount the lower limit on the LSP mass.

\subsection{Constraints from Higgs boson searches}

To derive the results discussed in this section, the analysis presented in Ref. [3] was updated with the inclusion of the results of the ALEPH searches for Higgs bosons [4]. For a given set of $\tan \beta, m_{0}$ and $M_{2}$ values, the largest predicted Higgs boson mass is determined with a large value for $m_{\mathrm{A}}, 2 \mathrm{TeV} / c^{2}$, and maximal effect from stop mixing by varying the combination $\tilde{A}_{\mathrm{t}}=A_{\mathrm{t}}-\mu \cot \beta$. For large $m_{\mathrm{A}}$, the lighter scalar neutral Higgs boson becomes standard-model like. It is therefore sufficient to compare the largest predicted $m_{\mathrm{h}}$ to the standard model Higgs boson mass lower limit of $107.7 \mathrm{GeV} / c^{2}$, as determined in Ref. «. The loophole which might have arisen from possible Higgs boson decays into stop pairs [3] is avoided by the recent parameter-independent stop mass lower limit of $63 \mathrm{GeV} / c^{2}$ [25. The lower limit on $M_{2}$ obtained in this way is displayed in Fig. 5 as a function of $\tan \beta$, for various values of $m_{0}$. As expected, the limit is strongest for low values of $\tan \beta$ and $m_{0}$. For instance, $M_{2}$ is larger than $110 \mathrm{GeV} / c^{2}$ for $\tan \beta=2.5$ and $m_{0}=200 \mathrm{GeV} / c^{2}$.

Although weaker as $m_{0}$ increases, the constraints on $M_{2}$ are still sufficient to improve on the LSP mass lower limit discussed in Section 3.4. For instance, for $m_{0}=1 \mathrm{TeV} / c^{2}$ the limit is $47.5 \mathrm{GeV} / c^{2}$ (obtained for $\tan \beta \simeq 2$ ); it is $46 \mathrm{GeV} / c^{2}$ for $m_{0}=2 \mathrm{TeV} / c^{2}$ (obtained for $\tan \beta \simeq 1.8$ ).

In the small $m_{0}$ regime, the LSP mass lower limit discussed in Section 3.4 depends on the extent to which the slepton searches cover the so-called corridor, a subset of model parameters giving sneutrinos almost degenerate in mass with the lightest charginos. The constraints from Higgs boson searches provide additional coverage of the corridor at small 
$\tan \beta$, as depicted in Fig. 6. While for $\tan \beta>3.5$ the Higgs boson constraints do not bring any improvement, the lower limit on $m_{\chi_{1}^{0}}$ set in the corridor by Higgs boson searches is larger than that set by slepton searches for $2.85<\tan \beta<3.5$.

For $\tan \beta<2.85$, the limit on $m_{\chi_{1}^{0}}$ set in the corridor by Higgs boson searches is no longer the absolute limit, since it exceeds that obtained from chargino searches for large $m_{0}$ values. This latter limit might degrade as $m_{0}$ is reduced, but the Higgs boson searches confine the charginos more and more in the higgsino region (Fig. 5), thus rendering the deleterious influence of light sleptons and light sneutrinos less and less important. As a result, the limit from chargino searches at large $m_{0}$ and $\tan \beta<2.85$ remains robust for any smaller $m_{0}$ value, and is even superseded by the limit arising from Higgs boson searches for $\tan \beta<1.95$.

The results obtained with the constraints from Higgs boson searches are however quite sensitive to the top quark mass. A value of $175 \mathrm{GeV} / c^{2}$ was used to derive the results reported in this section, i.e., an LSP mass lower limit of $38 \mathrm{GeV} / c^{2}$, irrespective of $\tan \beta$, and of $45 \mathrm{GeV} / c^{2}$ for $\tan \beta<3$ (Fig. 6). If a value of $180 \mathrm{GeV} / c^{2}$ is chosen instead, the absolute lower limit of $38 \mathrm{GeV} / c^{2}$ still holds, but the limit for $\tan \beta<3$ is reduced to $40 \mathrm{GeV} / c^{2}$. If, in addition, a value as large as $2 \mathrm{TeV} / c^{2}$ (instead of $\left.1 \mathrm{TeV} / c^{2}\right)$ is allowed for $m_{0}$, the Higgs boson searches no longer improve at low $\tan \beta$ on the limit of $37.2 \mathrm{GeV} / c^{2}$ deduced from the chargino and neutralino searches for large sfermion masses.

\subsection{Interpretation in Minimal Supergravity}

The interplay among the searches for sleptons, charginos and Higgs bosons, and the Z width measurement at LEP1 [26], is shown in Fig. 7 as exclusion domains in the $\left(m_{0}, m_{1 / 2}\right)$ plane for $\tan \beta=5$ and 10 , for $\mu<0$ and $\mu>0$, and for $A_{0}=0$; the top quark mass was set to $175 \mathrm{GeV} / c^{2}$.

The scan of the $\left(m_{0}, m_{1 / 2}\right)$ plane allows constraints to be derived on the mass of the lightest neutralino. As already noticed in Ref. [27], it is possible to find configurations where the analyses discussed in this letter lose their sensitivity; these loopholes open up when the lightest $\widetilde{\tau}$ is almost degenerate with the $\chi_{1}^{0}$. To obtain the results reported in this section, these configurations were avoided by the requirement that $m_{\tilde{\tau}}-m_{\chi_{1}^{0}}$ be larger than $5 \mathrm{GeV} / c^{2}$.

The lower limit on $m_{\chi_{1}^{0}}$ as a function of $\tan \beta$ is shown in Fig. 8 for $A_{0}=0$ and for both $\mu<0$ and $\mu>0$. There is little structure in the limit for both signs of $\mu$. Small $\tan \beta$ values are excluded by the negative result of Higgs boson searches, whereas for larger $\tan \beta$ values the limit is determined mostly by chargino searches. The lowest allowed value for $m_{\chi_{1}^{0}}$ is always found at large $m_{0}$; it has been calculated for $m_{0}=1 \mathrm{TeV} / c^{2}$. Altogether, a $\chi_{1}^{0}$ mass lower limit of $49 \mathrm{GeV} / c^{2}$ is set for $A_{0}=0$. It is reached for $\tan \beta \sim 4.5$ and $\mu<0$.

The impact of a non-vanishing $A_{0}$ value was studied by scanning the range allowed by theoretical constraints and by stop searches. The lower limit on $m_{\chi_{1}^{0}}$ as a function of $\tan \beta$ obtained from the $A_{0}$ scan is also shown in Fig. 8. Altogether, the $\chi_{1}^{0}$ mass lower limit decreases to $48 \mathrm{GeV} / c^{2}$, a value reached for $\tan \beta \sim 4.5$ and $\mu<0$. The lower limit 
on $m_{\chi_{1}^{0}}$ is therefore substantially improved when the additional constraints of minimal supergravity are considered.

\section{Conclusions}

The previously published ALEPH searches for sleptons, squarks, charginos and neutralinos have been updated with the data collected during 1998 and 1999 at centre-of-mass energies up to $201.6 \mathrm{GeV}$, corresponding to an overall integrated luminosity of about $410 \mathrm{pb}^{-1}$. In all topologies, the numbers of candidate events observed are consistent with the background expected from standard processes. When interpreted in the framework of the MSSM, this negative outcome allows improved 95\% C.L. lower limits on slepton and squark masses to be set as summarized in Table 4.

Table 4: Lower limits at 95\% C.L. on squark and slepton masses. For selectrons and sleptons, $\tan \beta=2, \mu=-200 \mathrm{GeV} / c^{2}$. For sleptons, a common mass at the GUT scale is assumed. All masses and mass differences are in $\mathrm{GeV} / c^{2}$.

\begin{tabular}{|c|c|c|}
\hline Particle & Limit & Conditions of validity \\
\hline selectron & 92 & $\Delta M>10$ \\
\hline smuon & 85 & $\Delta M>10, \tilde{\mu} \rightarrow \mu \chi_{1}^{0}$ \\
\hline \multirow[t]{2}{*}{ stau } & 68 & $\begin{array}{c}\Delta M>10, \tilde{\tau} \rightarrow \tau \chi_{1}^{0} \\
\text { worst case mixing }\end{array}$ \\
\hline & 70 & $\Delta M>10, \tilde{\tau} \rightarrow \tau \chi_{1}^{0}, \tilde{\tau}_{\mathrm{R}}$ \\
\hline \multirow[t]{2}{*}{ slepton } & 93 & $\Delta M>10$ \\
\hline & 70 & any $\Delta M$ \\
\hline \multirow[t]{2}{*}{ stop } & 83 & $\overline{\widetilde{\mathrm{t}}} \rightarrow \mathrm{c} \chi_{1}^{0}, 6<\Delta M<40$ \\
\hline & 88 & $\widetilde{\mathrm{t}} \rightarrow \mathrm{b} \ell \widetilde{\nu}, \Delta M>10$ \\
\hline sbottom & 91 & $\widetilde{\mathrm{b}} \rightarrow \mathrm{b} \chi_{1}^{0}, \Delta M>8, \tilde{\mathrm{b}}_{\mathrm{L}}$ \\
\hline $\begin{array}{l}\text { degenerate } \\
\text { squarks }\end{array}$ & 97 & $\begin{array}{c}\tilde{\mathrm{q}} \rightarrow \mathrm{q} \chi_{1}^{0}, \Delta M>6 \\
\tan \beta=4, \mu=-400\end{array}$ \\
\hline
\end{tabular}

Chargino pair production and neutralino associated production are excluded up to the kinematic limit in a significant fraction of the MSSM parameter space. For $m_{0}>$ $500 \mathrm{GeV} / c^{2}$, a lower limit on $m_{\chi_{1}^{0}}$ of $37 \mathrm{GeV} / c^{2}$ is obtained, independent of $\tan \beta$. It has been verified that this limit remains valid for all $m_{0}$ values if the constraints from slepton searches are taken into account, for a negligible mixing in the stau sector.

The negative results of the searches for Higgs bosons reported in Ref. [4] further constrain the MSSM parameter space. In particular, the lower limit on the $\chi_{1}^{0}$ mass is increased to $45 \mathrm{GeV} / c^{2}$ for $\tan \beta<3$ and to $38 \mathrm{GeV} / c^{2}$ for any $\tan \beta$, assuming $m_{\mathrm{t}}=175 \mathrm{GeV} / c^{2}$ and $m_{0}<1 \mathrm{TeV} / c^{2}$. 
The results have also been interpreted in the framework of minimal supergravity, including one-loop radiative corrections in the calculation of chargino and neutralino masses. The domains excluded in the $\left(m_{0}, m_{1 / 2}\right)$ plane are significantly larger than those reported by the D0 collaboration from the negative results of searches for gluinos and squarks at the Tevatron 28]. The resulting lower limit on $m_{\chi_{1}^{0}}$ is $49 \mathrm{GeV} / c^{2}$ for $A_{0}=0$ and $m_{0}<1 \mathrm{TeV} / c^{2}$. It is reduced to $48 \mathrm{GeV} / c^{2}$ when $A_{0}$ is varied, provided that the parameter configurations leading to $m_{\tilde{\tau}}-m_{\chi_{1}^{0}}<5 \mathrm{GeV} / c^{2}$ are ignored.

These results improve significantly on those obtained at lower energies by ALEPH [3] and by the L3 [29], OPAL [30] and DELPHI [31] collaborations. Less general searches for associated production of charginos and neutralinos have also been reported by the CDF [32] and D0 [33] collaborations.

\section{Acknowledgements}

It is a pleasure to congratulate our colleagues from the accelerator divisions for the successful operation of LEP2. We are indebted to the engineers and technicians in all our institutions for their contribution to the excellent performance of ALEPH. Those of us from non-member states wish to thank CERN for its hospitality and support.

\section{References}

[1] ALEPH Coll., "Search for sleptons and squarks in $e^{+} e^{-}$collisions at $\sqrt{s} \sim 189$ GeV", Phys. Lett. B 469 (1999) 303.

[2] ALEPH Coll., "Searches for Charginos and Neutralinos in $e^{+} e^{-}$Collisions at $\sqrt{s}=$ 161 and 172 GeV", Eur. Phys. J. C2 (1998) 417.

[3] ALEPH Coll., "Search for Charginos and Neutralinos in $e^{+} e^{-}$Collisions at Centreof-Mass Energies near 183 GeV and Constraints on the MSSM Parameter Space", Eur. Phys. J. C 11 (1999) 193.

[4] ALEPH Coll., "Searches for neutral Higgs bosons in $e^{+} e^{-}$collisions at centre-of-mass energies from 192 to 202 GeV", CERN-EP/2000-131, submitted to Phys. Lett. B.

[5] M. Carena et al., Nucl. Phys. B 580 (2000) 29.

[6] P. Janot, "The HZHA generator", in "Physics at LEP2", Eds. G. Altarelli, T. Sjöstrand and F. Zwirner, CERN 96-01 (1996) Vol. 2., p. 309; For a description of the updates and for the code, see: http://alephwww.cern.ch/ janot/Generators.html.

[7] F.E. Paige, S.D. Protopopescu, H. Baer, and X. Tata, "ISAJET 7.51- A Monte Carlo Event Generator for $p p, p \bar{p}$ and $e^{+} e^{-}$Reactions"; documentation and code available from ftp : //penguin.phy.bnl.gov/pub/isajet/.

[8] J. Ellis et al., Phys. Rev. D58 (1998) 095002. 
[9] ALEPH Coll., "ALEPH: a detector for electron-positron annihilations at LEP", Nucl. Instrum. and Methods A294 (1990) 121; D. Creanza et al., "The new ALEPH silicon vertex detector", Nucl. Instrum. and Methods A409 (1998) 157.

[10] ALEPH Coll., "Performance of the ALEPH detector at LEP", Nucl. Instrum. and Methods A360 (1995) 481.

[11] S. Katsanevas and P. Morawitz, Comp. Phys. Commun. 112 (1998) 227.

[12] S. Jadach et al., Phys. Lett. B 390 (1997) 298.

[13] S. Jadach et al., Comp. Phys. Commun. 36 (1985) 191.

[14] J.A.M. Vermaseren, in Proceedings of the IVth international Workshop on Gamma Gamma Interactions, Eds. G. Cochard and P. Kessler, Springer Verlag, 1980;

ALEPH Coll., "An experimental study of $\gamma \gamma \rightarrow$ hadrons at LEP", Phys. Lett. B313 (1993) 509.

[15] R. Engel, Z. Phys. C66 (1995) 203; R. Engel and J. Ranft, Phys. Rev. D54 (1996) 4144.

[16] M. Skrzypek et al., Comp. Phys. Commun. 94 (1996) 216.

[17] J. Fujimoto et al., Comp. Phys. Commun. 100 (1997) 128.

[18] This generator is based on the differential cross section by S. Ambrosanio and B. Mele published in Nucl. Phys. B 374 (1992) 3.

[19] T. Sjöstrand, Comp. Phys. Commun. 82 (1994) 74; CERN-TH 7112/93 (1993, revised August 1994).

[20] ALEPH Coll., "Scalar quark searches in $e^{+} e^{-}$collisions at $\sqrt{s}=181-184$ GeV", Phys. Lett. B 434 (1998) 189.

[21] R.D. Cousins and V.L. Highland, Nucl. Instrum. and Methods A 320 (1992) 331.

[22] R. M. Barnett et al. (Particle Data Group), Phys. Rev. D 54 (1996) 1.

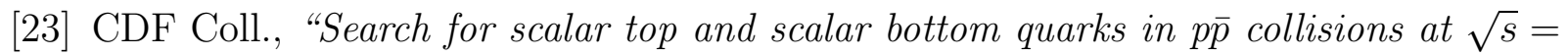
1.8 TeV", FERMILAB-PUB-99/311-E; "Search for Scalar Top Quark Production in $p \bar{p}$ Collisions at $\sqrt{s}=1.8$ TeV", FERMILAB-PUB-99/340-E; "Search for gluinos and squarks at the Fermilab Tevatron collider", Phys. Rev. D 56 (1997) 1357.

[24] D0 Coll., "Search for Squarks and Gluinos in $p \bar{p}$ Collisions at $\sqrt{s}=1.8$ TeV", Phys. Rev. Lett. 75 (1995) 618; "Search for Squarks and Gluinos in Events Containing Jets and Large Imbalance in Transverse Energy", Phys. Rev. Lett. 83, (1999) 4937; "Search for Bottom Squarks in $\mathrm{p} \overline{\mathrm{p}}$ Collisions at $\sqrt{s}=1.8$ TeV", Phys. Rev. D Rapid Comm. 60, (1999) 031101.

[25] ALEPH Coll., "Search for a scalar top almost degenerate with the lightest neutralino in $e^{+} e^{-}$collisions at $\sqrt{s}$ up to 202 GeV", CERN-EP/2000-085, submitted to Phys. Lett. B. 
[26] ALEPH Coll., "Measurement of the Z Resonance Parameters at LEP", Eur. Phys. J. C 14 (2000) 1.

[27] LEP SUSY Working Group, ALEPH, DELPHI, L3 and OPAL experiments, "Interpretation in Minimal SUGRA and limit on the LSP mass", LEPSUSYWG/99$03.1 ;$ http://lepsusy.web.cern.ch/lepsusy/.

[28] D0 Coll., "Search for Squarks and Gluinos in Events Containing Jets and a Large Imbalance in Transverse Energy", Phys. Rev. Lett. 83 (1999) 4937; "A Search for Dilepton Signature from Minimal Low-energy Supergravity $\mathrm{p} \overline{\mathrm{p}}$ Collisions at 1800 GeV", Fermilab-Pub-00/042-E.

[29] L3 Coll., "Searches for Scalar Leptons in $e^{+} e^{-}$collisions at $\sqrt{s}=189$ GeV", Phys. Lett. B 471 (1999) 280; "Searches for Scalar Quarks in $e^{+} e^{-}$Interactions at $\sqrt{s}=189$ GeV", Phys. Lett. B 471 (1999) 308; "Search for Charginos and Neutralinos in $e^{+} e^{-}$collisions at $\sqrt{s}=189$ GeV", Phys. Lett. B 472 (2000) 420;

[30] OPAL Coll., "Search for Scalar Top and Scalar Bottom Quarks at $E_{C M}=189$ GeV at LEP", Phys. Lett. B 456 (1999) 95; "Search for Anomalous Production of Acoplanar Di-lepton Events in $e^{+} e^{-}$collisions at $\sqrt{s}=183$ and 189 GeV", Eur. Phys. J. C 14 (2000) 51; "Search for Chargino and Neutralino Production at $\sqrt{s}=189$ GeV at LEP", CERN-EP/99-123.

[31] DELPHI Coll., "Search for Scalar Fermions and Long-Lived Scalar Leptons at Centreof-Mass Energies of 130 GeV to 172 GeV", Eur. Phys. J. C 6 (1999) 385; "Search for charginos, neutralinos and gravitinos in $e^{+} e^{-}$interactions at $\sqrt{s}=183 \mathrm{GeV}$ ", Phys. Lett. B 446 (1999) 75; "Search for charginos in $\mathrm{e}^{+} \mathrm{e}^{-}$interactions at $\sqrt{s}=$ 189 GeV", Phys. Lett. B 479 (2000) 129; "Search for supersymmetric partners of top and bottom quarks at $\sqrt{s}=189$ GeV", CERN-EP/2000-095.

[32] CDF Coll., "Search for Chargino-Neutralino Associated Production at the Fermilab Tevatron Collider", Phys. Rev. Lett. 80 (1998) 5275.

[33] D0 Coll., "Search for Trilepton Signatures from Associated Gaugino Pair Production", Phys. Rev. Lett. 80 (1998) 1591. 


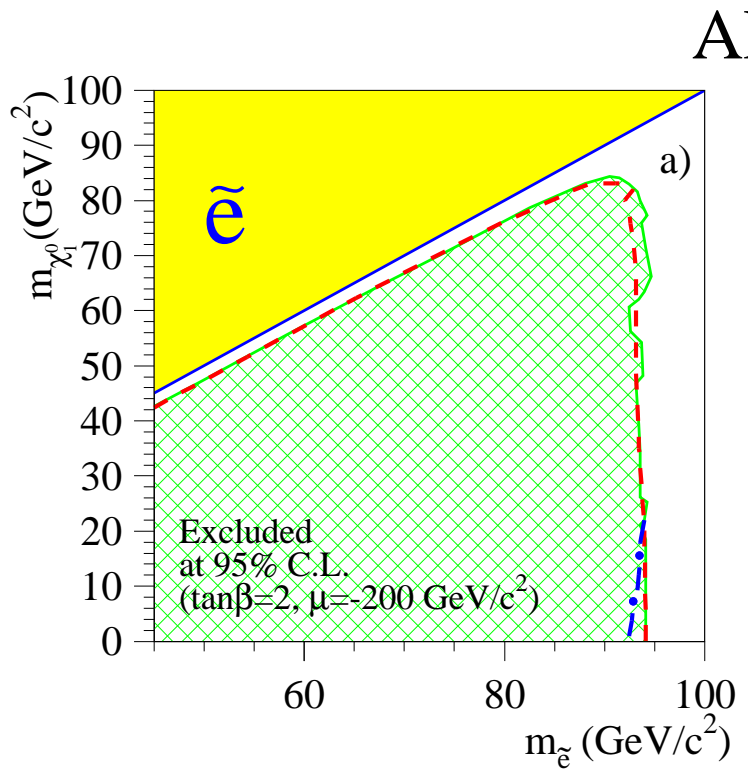

ALEPH
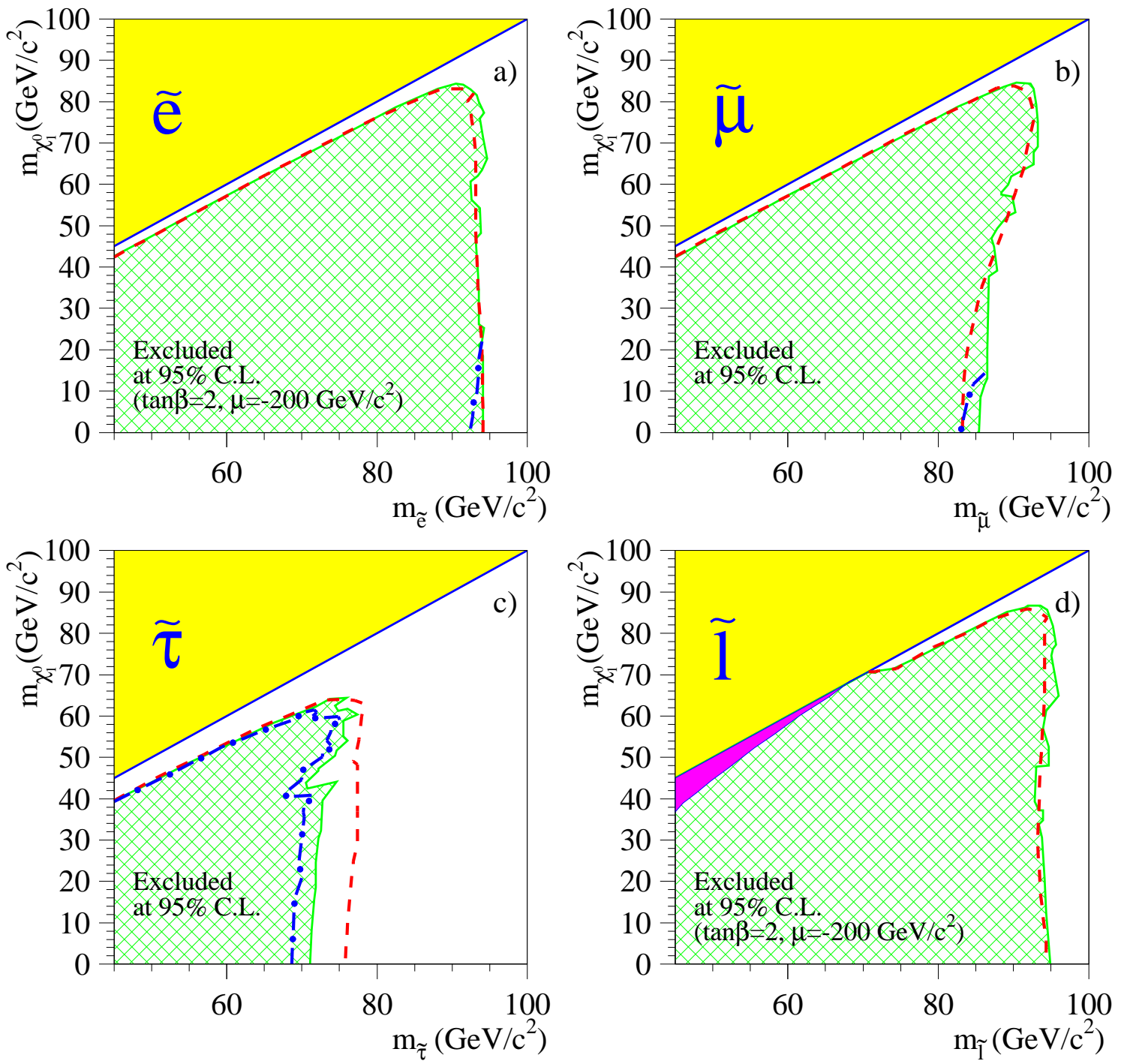

Figure 1: Excluded regions at $95 \%$ C.L. in the $m_{\tilde{\ell}_{\mathrm{R}}}$ vs $m_{\chi_{1}^{0}}$ plane from slepton searches assuming $\operatorname{BR}\left(\tilde{\ell} \rightarrow \ell \chi_{1}^{0}\right)=100 \%$ (cross-hatched regions); the dashed curves show the expected exclusion under the same assumptions. The dot-dashed curves in a) and $b$ ) show the effect of cascade decays for $\tan \beta=2$ and $\mu=-200 \mathrm{GeV} / c^{2}$, assuming zero efficiency for those decays. The dot-dashed curve in $c$ ) shows the limit in the case of minimal $\tilde{\tau}_{1} \tilde{\tau}_{1} \mathrm{Z}$ coupling. In $d$ ), the effect of cascade decays is included. The dark shaded region is not accessible because the common scalar mass at the GUT scale becomes unphysical. In all the plots, the light shaded region corresponds to the $m_{\tilde{\ell}}<m_{\chi_{1}^{0}}$ disallowed region. 


\section{ALEPH}
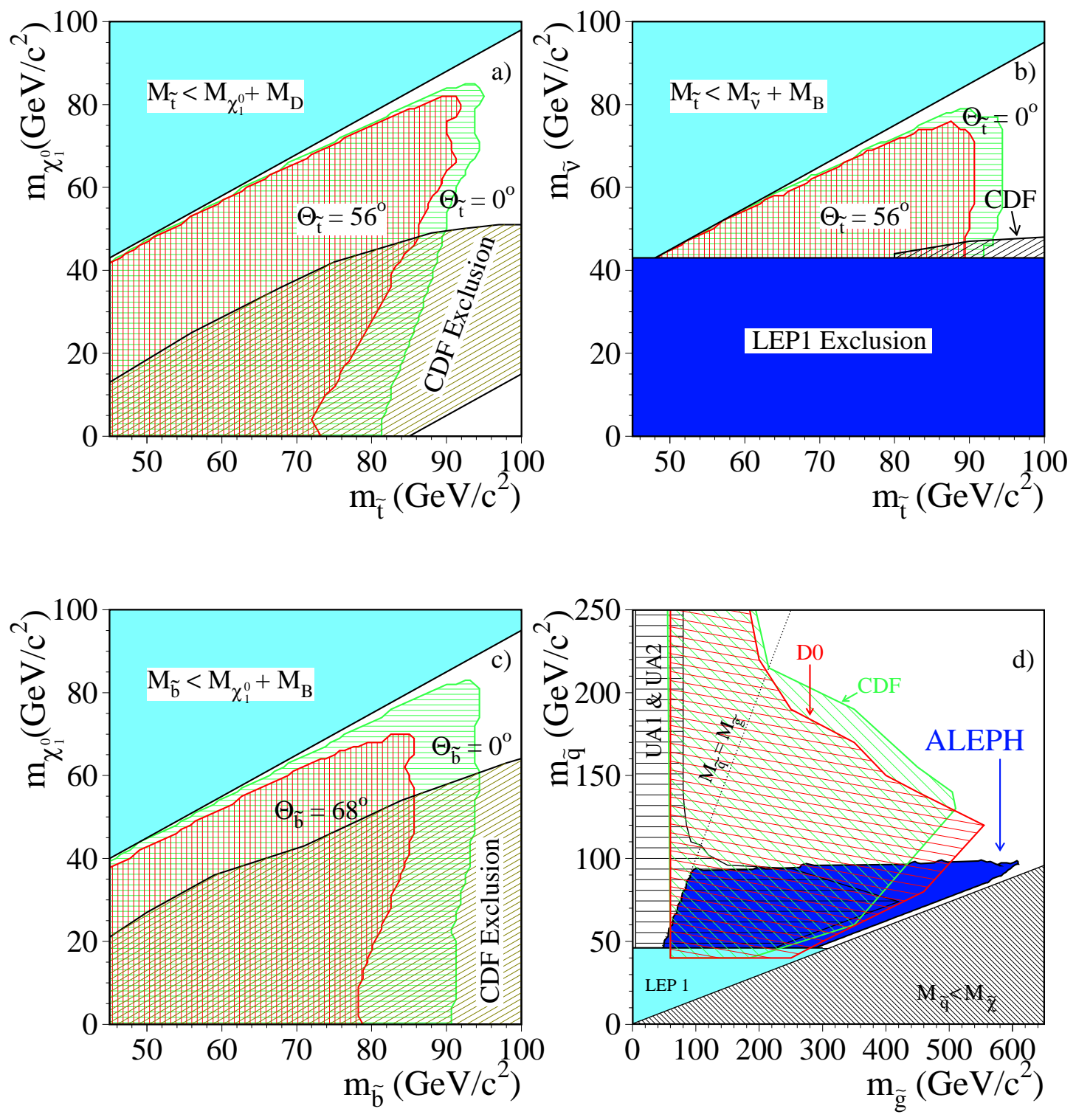

Figure 2: Excluded regions at $95 \%$ C.L. from squark searches: a) in the $\left(m_{\chi_{1}^{0}}, m_{\widetilde{t}}\right)$ plane assuming a dominant $\widetilde{\mathrm{t}} \rightarrow \mathrm{c} \chi_{1}^{0}$ decay; $\left.b\right)$ in the $\left(m_{\tilde{\nu}}, m_{\tilde{\mathrm{t}}}\right)$ plane for a dominant $\widetilde{\mathrm{t}} \rightarrow \mathrm{b} \ell \tilde{\nu}$ decay and equal branching fractions into e, $\mu$, and $\tau ; c)$ in the $\left(m_{\chi_{1}^{0}}, m_{\widetilde{\mathrm{b}}}\right)$ plane for a dominant $\widetilde{\mathrm{b}} \rightarrow \mathrm{b} \chi_{1}^{0}$ decay; $d)$ in the plane $\left(m_{\widetilde{\mathrm{g}}}, m_{\widetilde{\mathrm{q}}}\right)$ in the case of five mass degenerate $\widetilde{\mathrm{q}}$ flavours, $\tan \beta=4$ and $\mu$ $=-400 \mathrm{GeV} / c^{2}$. The hadron collider results are also shown for comparison. 
ALEPH
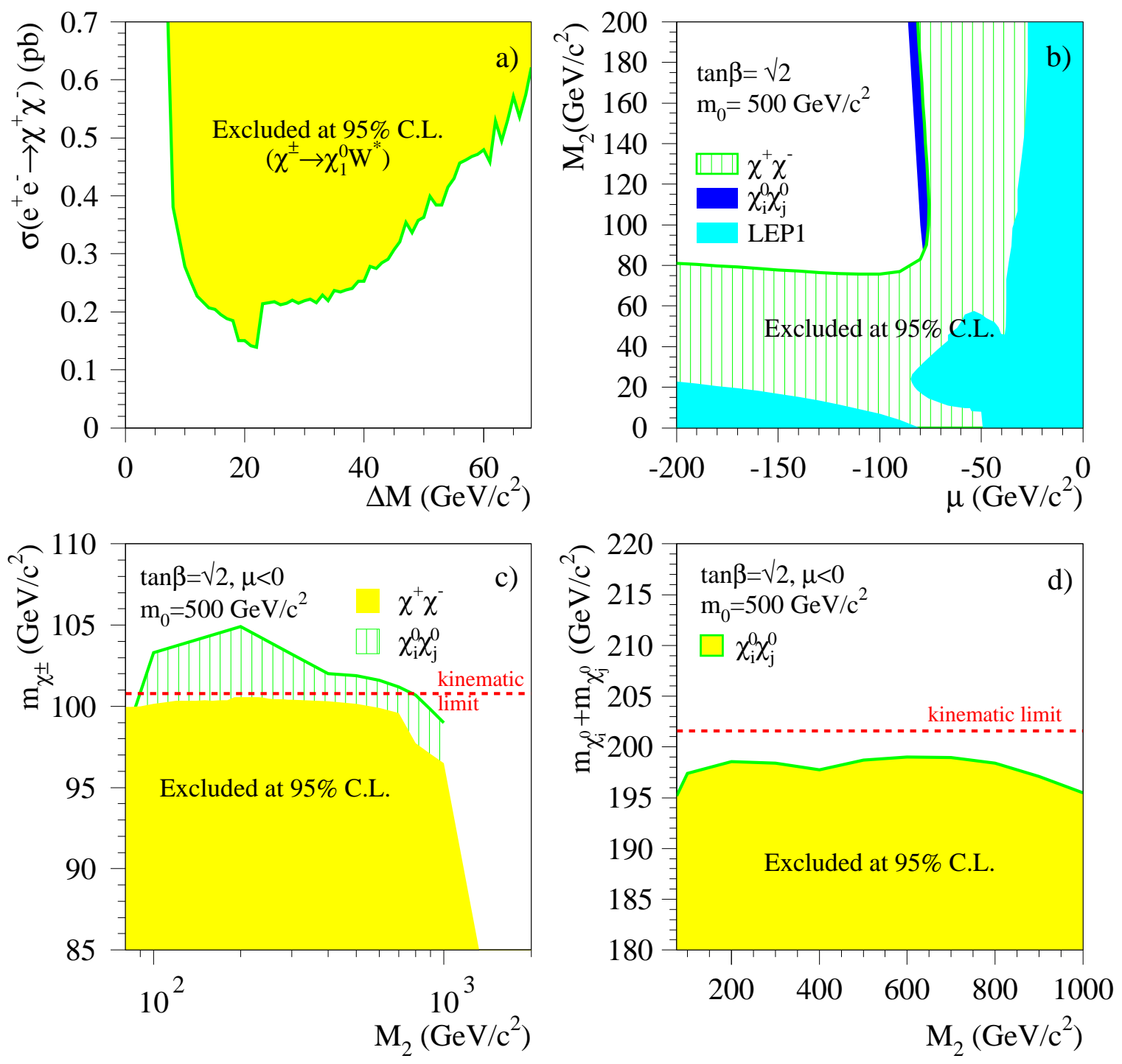

Figure 3: Gaugino searches: a) upper limit on the chargino pair production cross section for chargino masses close to the kinematic limit at $\sqrt{s}=201.6 \mathrm{GeV}$; b) excluded domains in the $\left(\mu, M_{2}\right)$ plane of the MSSM; $c$ ) chargino mass lower limit in the higgsino region; $d$ ) lower limit on the sum of the masses of two neutralinos produced with the largest cross section $\left(\chi_{1}^{0} \chi_{2}^{0}\right.$ for large $M_{2}$ and mainly $\chi_{1}^{0} \chi_{3}^{0}$ for small $M_{2}$ ). In b, c, and d, it is assumed that $m_{0}=500 \mathrm{GeV} / c^{2}$, $\mu<0$ and $\tan \beta=\sqrt{2}$. 


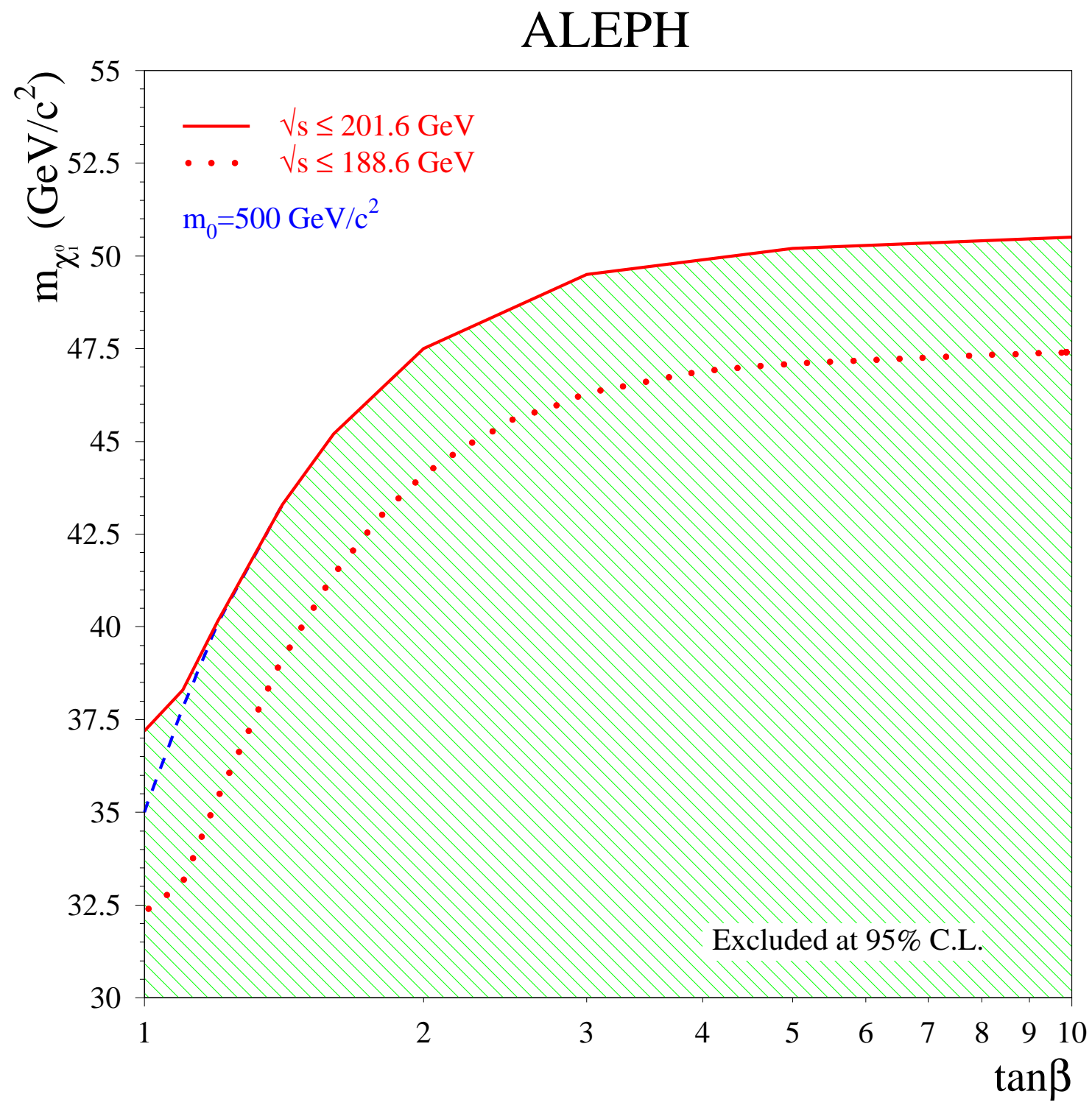

Figure 4: The $95 \%$ C.L. lower limit on the mass of the lightest neutralino as a function of $\tan \beta$ for $m_{0}=500 \mathrm{GeV} / c^{2}$. The dashed line indicates the limit obtained without the constraints from the searches for neutralinos. The result obtained with the data collected at $\sqrt{s}$ up to $189 \mathrm{GeV}$ is shown for comparison. Higher $m_{0}$ values give similar limits. 


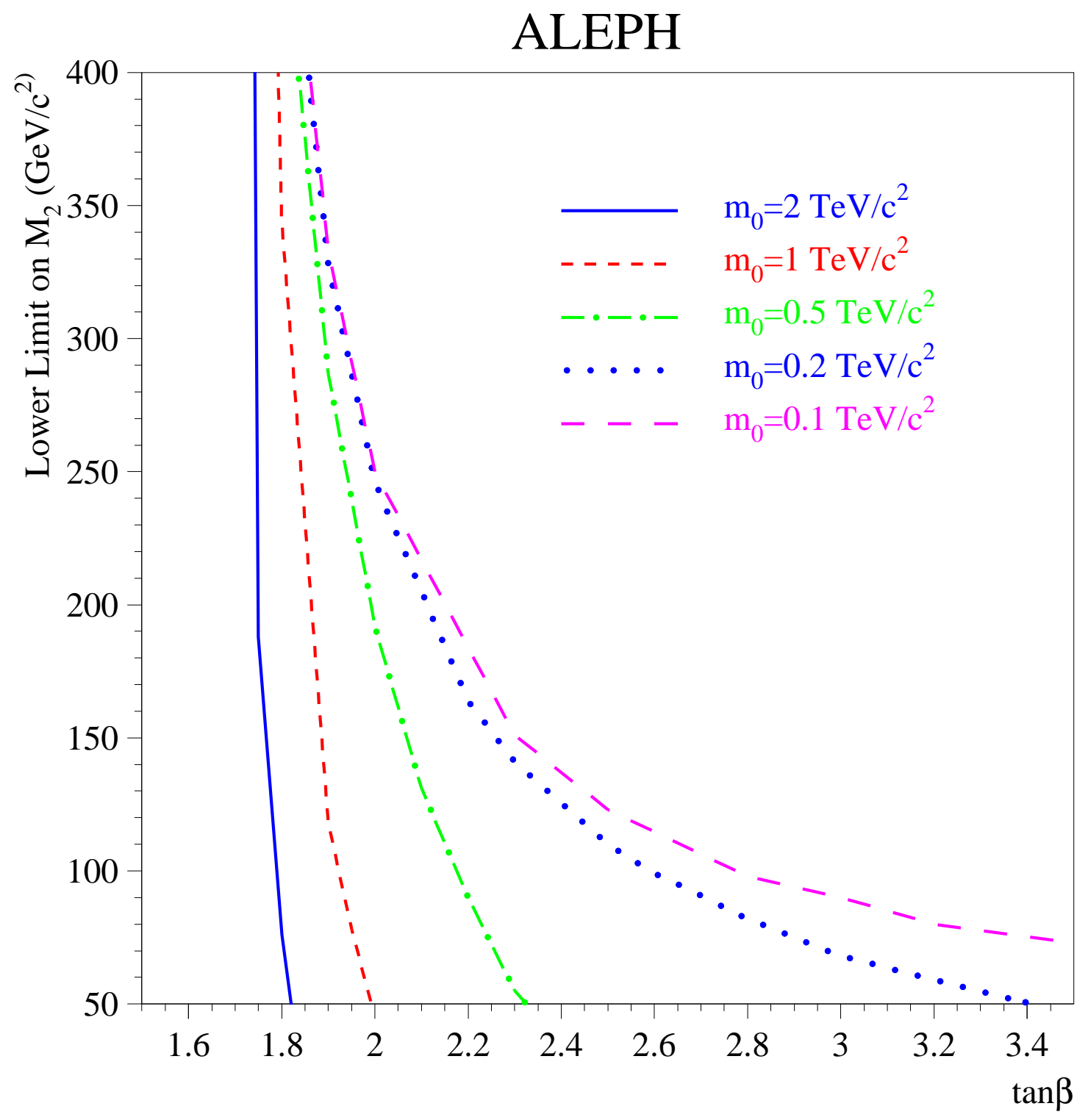

Figure 5: Lower limit on $M_{2}$ as a function of $\tan \beta$, as determined from the result of Higgs boson searches, for five values of $m_{0}$. 


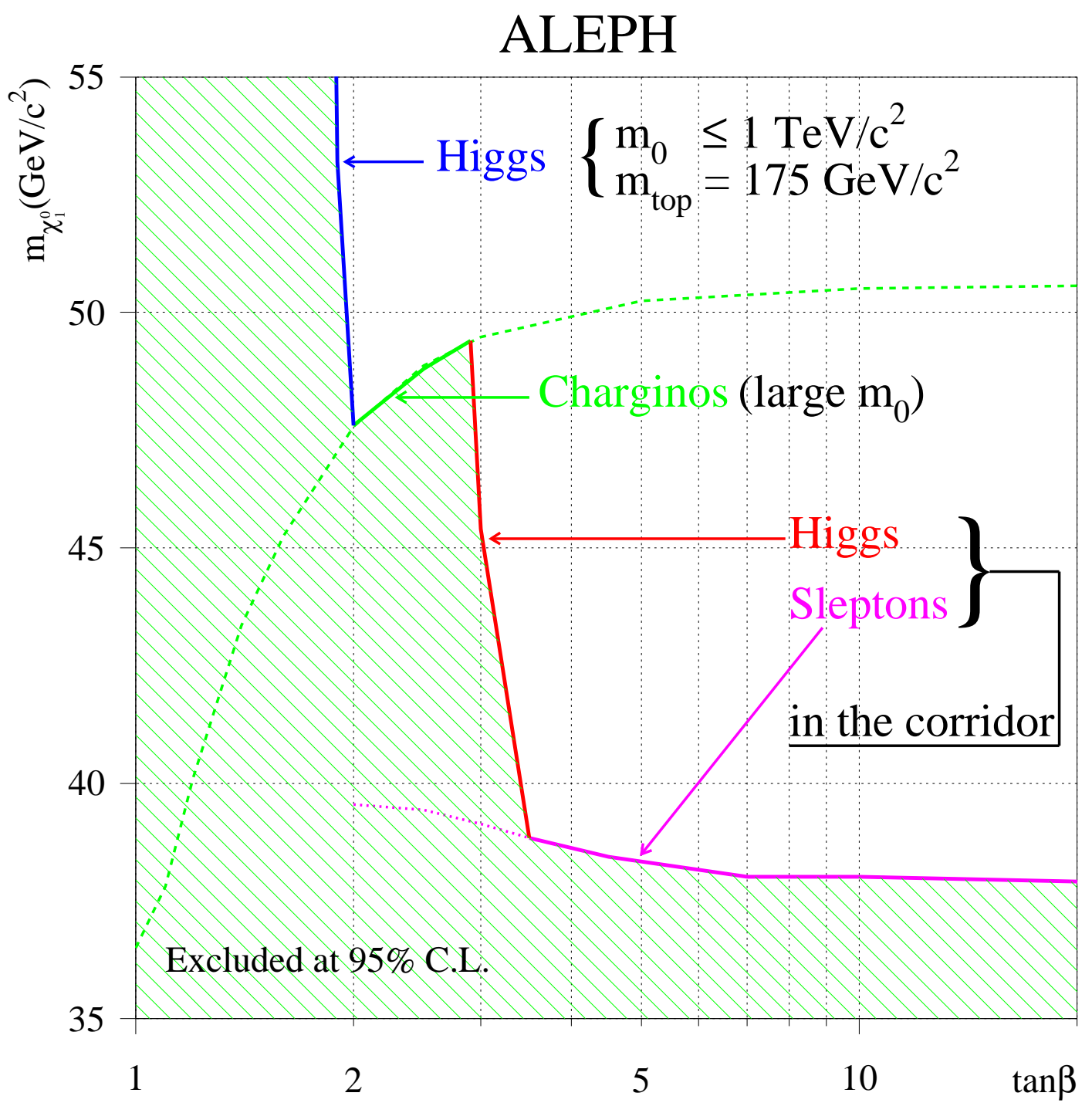

Figure 6: Lower limit on $m_{\chi_{1}^{0}}$ as a function of $\tan \beta$, from, right to left, slepton searches in the corridor $\left(m_{\chi^{ \pm}} \simeq m_{\tilde{\nu}}\right)$, Higgs boson searches in the corridor, chargino searches for large sfermion masses, and Higgs boson searches. The limits from Higgs boson searches are valid for $m_{\mathrm{t}}=175 \mathrm{GeV} / c^{2}$ and for $m_{0}$ not exceeding $1 \mathrm{TeV} / c^{2}$. The dashed curve indicates the limit from chargino and neutralino searches for large $m_{0}$. The dotted curve shows the limit in the corridor reached without the Higgs boson searches. 

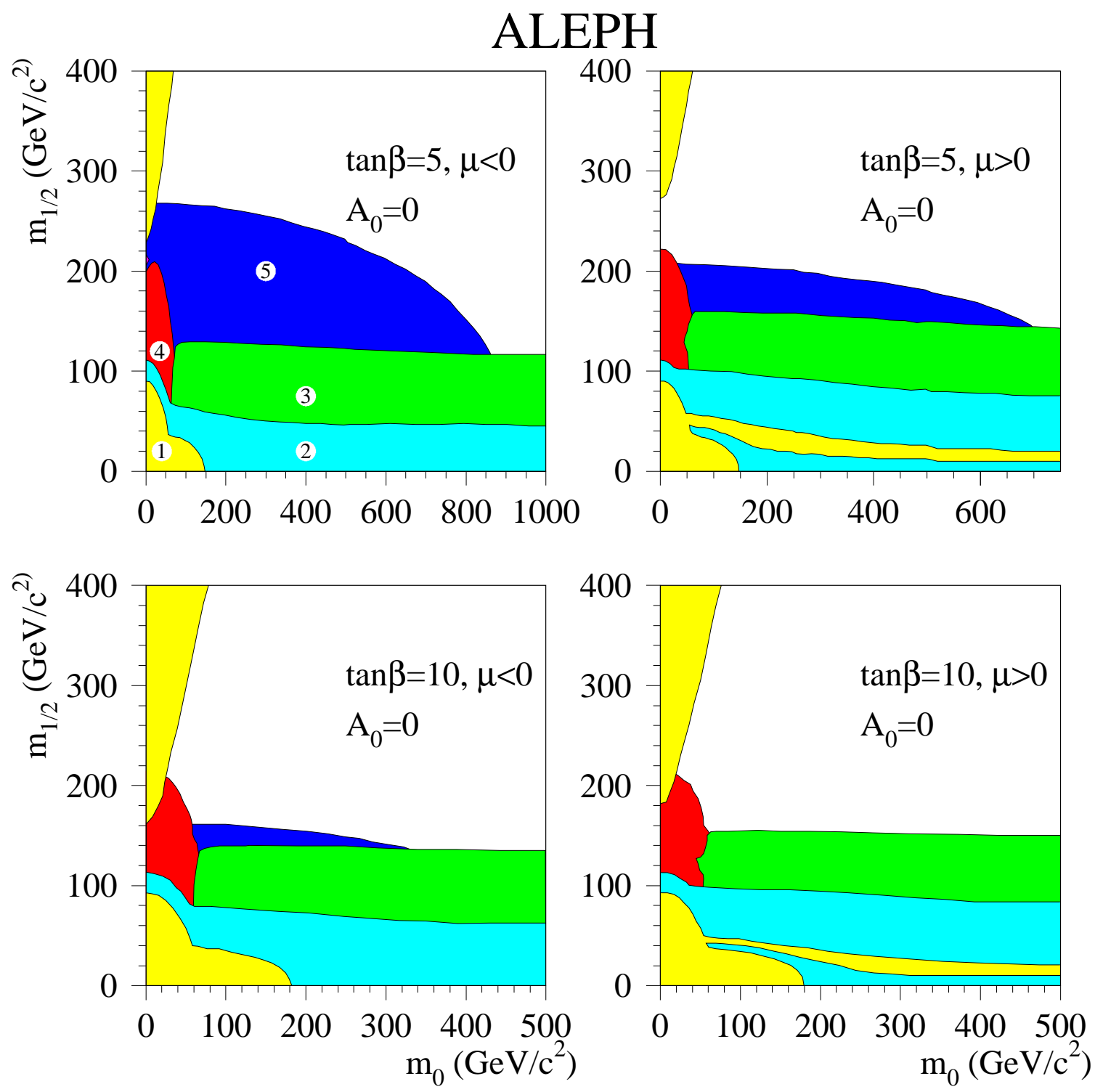

Figure 7: Minimal supergravity scenario: domains of the $\left(m_{0}, m_{1 / 2}\right)$ plane excluded for $\tan \beta=5$ and 10 and for $A_{0}=0$. Region 1 is theoretically forbidden. The other regions are excluded by the $\mathrm{Z}$ width measurement at LEP1 (2), chargino (3), slepton (4) searches and Higgs boson searches at $\sqrt{s} \leq 201.6 \mathrm{GeV}(5)$. 

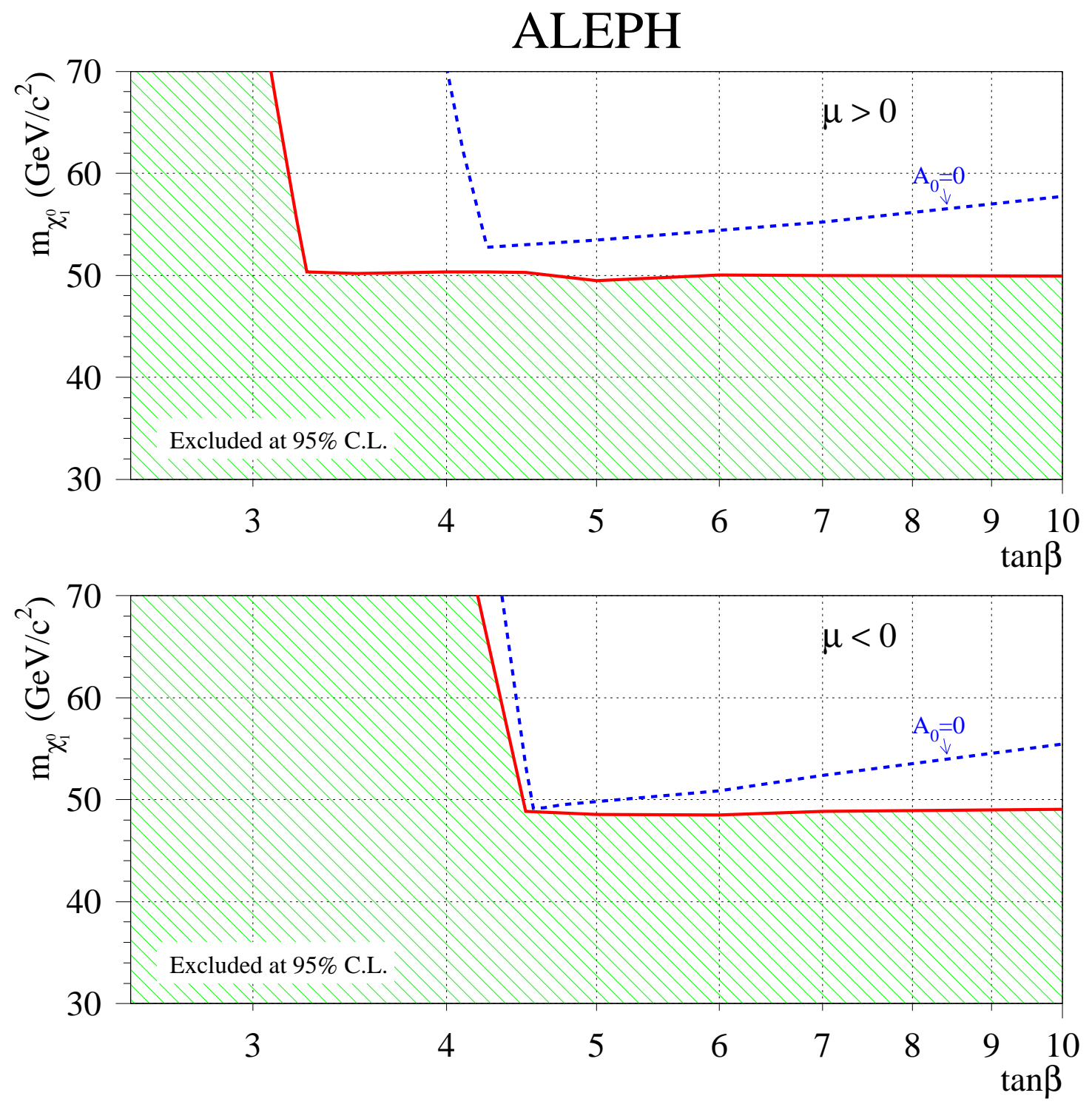

Figure 8: Minimal supergravity scenario: lower limit on the LSP mass as a function of $\tan \beta$. The dashed line shows the result obtained with $A_{0}=0$. 